\title{
Optical Postsynaptic Measurement of Vesicle Release Rates for Hippocampal Synapses Undergoing Asynchronous Release during Train Stimulation
}

\author{
Yo Otsu ${ }^{1,2}$ and Timothy H. Murphy ${ }^{1,2,3}$ \\ ${ }^{1}$ Kinsmen Laboratory and Brain Research Center, Departments of ${ }^{2}$ Psychiatry and ${ }^{3}$ Physiology, University of British Columbia, Vancouver, British \\ Columbia, V6T 1 Z3 Canada
}

\begin{abstract}
Developing hippocampal neurons in microisland culture were found to undergo rapid depression of excitatory synaptic activity caused by consumption of their readily releasable pool (RRP) of vesicles in response to $20 \mathrm{~Hz}$ trains of stimulation. Associated with depression was a switch to an asynchronous release mode that maintained transmission at a high steady-state rate equivalent to $\sim 2.1$ RRPs per second. We have applied postsynaptic $\mathrm{Ca}^{2+}$ imaging to directly monitor these asynchronous release events to estimate both the steady rate of transmitter release and the number of quanta within the RRP at individual hippocampal synapses. Based on the frequency of asynchronous release measured at individual synapses postsynaptically using $\mathrm{Ca}^{2+}$ imaging (5-17 sec after train stimulation) and with knowledge of the time course by which asynchronous release rates decay, we estimate that individual hippocampal synapses exhibit (in response to train stimulation) peak release rates of up to 21 quanta per second from an RRP that contains, on average, 10 quanta. Use-dependent block of evoked synaptic activity by MK-801 [(+)-5-methyl-10,11-dihydro-5H-dibenzo [a,d]cyclohepten-5,10-imine maleate] confirmed that synapses undergoing asynchronous release are not significantly different from the general population with regard to their composition of NMDA receptor and/or release probability. Given that high-frequency trains deplete the synapse of readily releasable quanta (and that these release rates can only be maintained for a few seconds), these high rates of asynchronous release likely reflect refilling of vesicles from a reserve pool and not necessarily the continuous action of a relatively slow clathrin- and endosomedependent process.
\end{abstract}

Key words: calcium; dendrite; desynchronization; imaging; NMDA receptor; quantal

\section{Introduction}

The response of CNS synapses to high-frequency stimulation is a function of the number of readily releasable quanta of the synapses and the probability of release, as well as their ability to refill and mobilize additional quanta (Rosenmund and Stevens, 1996; Dobrunz and Stevens, 1997; Wang and Kaczmarek, 1998; Aravanis et al., 2003; Gandhi and Stevens, 2003). The maximal rate at which a synapse refills itself is important because this will determine the frequency of stimulation that it can follow (Harata et al., 2001b). Given their small size and limited store of quanta, CNS synapses have evolved mechanisms distinct from their neuromuscular counterparts to rapidly cycle vesicles avoiding synaptic depression when driven at physiologically relevant frequencies (Harata et al., 2001b). Previous studies evaluating the response of CNS synapses to prolonged stimulation have revealed a rather wide range of recovery rates that are presumably related to un-

Received May 27, 2004; revised Aug. 31, 2004; accepted Sept. 2, 2004.

This study was supported by Canadian Institutes of Health Research (CIHR) Grant MT12675 to T.H.M. T.H.M. is a CIHR investigator and a Michael Smith Foundation for Health Research senior scholar. We thank Kerry Delaney, Vahid Shahrezaei, and Philippe Isope for helpful discussions and comments on a draft of this manuscript.

Correspondence should be addressed to Dr. T. H. Murphy, Department of Psychiatry, 4N1-2255 Wesbrook Mall, Vancouver, British Columbia, V6T 123 Canada. E-mail: thmurphy@interchange.ubc.ca.

DOI:10.1523/JNEUROSCI.2060-04.2004

Copyright $\odot 2004$ Society for Neuroscience $\quad$ 0270-6474/04/249076-11\$15.00/0 derlying refilling processes (Ryan et al., 1993; Rosenmund and Stevens, 1996; Klingauf et al., 1998; Pyle et al., 2000; Wesseling and Lo, 2002; Aravanis et al., 2003). Differences between studies may arise because some synapses use $\mathrm{Ca}^{2+}$-stimulated refilling that may be selectively engaged at particular stimulus frequencies (Stevens and Wesseling, 1998; Wang and Kaczmarek, 1998). For example, with high-frequency stimulation, quanta may be rapidly reused without having to undergo relatively slow endocytosis and recycling through endosomes, allowing synapses to exhibit high steady-state rates of transmitter release (Harata et al., 2001a,b). Although with lower-frequency stimulation the store of releasable vesicles of the synapses may suffice, and relatively slower recovery consistent with conventional clathrin-mediated endocytosis is observed (Sara et al., 2002). Some ambiguity may arise because imaging of quantal release using FM dyes or protein probes can be complicated by the nature of the recycling mechanism (Richards et al., 2000). In particular, not all presynaptic probes resolve rapid transient forms of vesicle turnover such as kiss-and-run exocytosis (Klingauf et al., 1998). In most previous studies, only synapse-specific measures of presynaptic vesicle turnover and not necessarily the postsynaptic effect of quantal release were directly monitored at individual synapses. Given that individual synapses may activity-dependently switch between multiple modes of release, it is unclear what impact this will have 
postsynaptically (Aravanis et al., 2003; Gandhi and Stevens, 2003). In cases in which the postsynaptic effects of individual quanta were monitored using $\mathrm{Ca}^{2+}$ imaging of NMDA receptor (NMDAR) activity, relatively low stimulus numbers and frequencies were used (Oertner et al., 2002; Emptage et al., 2003). Because intracellular $\mathrm{Ca}^{2+}$ is cleared relatively slowly (Holthoff et al., 2002; Sabatini et al., 2002) compared with the frequencies at which synapses operate physiologically, it has been difficult to view the release and postsynaptic action of individual quanta during train stimulation. Here we report a new approach in which postsynaptic quantal $\mathrm{Ca}^{2+}$ imaging or miniature synaptic $\mathrm{Ca}^{2+}$ transients (MSCTs) can be used to monitor trainstimulated asynchronous release. By knowing the time course by which asynchronous release rates decay, we estimate that peak rates of transmitter release at individual synapses average 21 quanta per second.

\section{Materials and Methods}

Hippocampal neuronal cultures. Hippocampal neurons were cultured on glial microislands as described previously (Bekkers and Stevens, 1991; Li et al., 2002; Otsu et al., 2004). Briefly, a 0.15\% agarose solution was spread uniformly on glass coverslips, and then a solution of poly-D-lysine $(0.5 \mathrm{mg} / \mathrm{ml})$ and collagen $(2 \mathrm{mg} / \mathrm{ml})$ was sprayed on the agarose background. Glial cells derived from rat cortex were then plated on the spots of adhesive surface created by the poly-D-lysine-collagen spray. After the glial microisland feeder layers were confluent, the CA1-CA3 regions of hippocampi were removed from postnatal day $0-3(\mathrm{P} 0-3)$ rats and then enzymatically ( $20 \mathrm{U} / \mathrm{ml}$ papain)-mechanically dissociated and plated on feeder layers. Cells were grown in a solution based on Neurobasal A medium supplemented with $2.5 \%$ fetal calf serum, 2\% B27, $20 \mathrm{~mm}$ glucose, and a $0.2 \%$ penicillin-streptomycin stock. Cultures were used for experiments $4-10 \mathrm{~d}$ after plating.

Electrophysiology. Cultures were superfused continuously with an extracellular recording solution containing (in $\mathrm{mm}$ ): $134 \mathrm{NaCl}, 2.5 \mathrm{KCl}, 3$ $\mathrm{CaCl}_{2}, 1 \mathrm{MgCl}_{2}, 0.34 \mathrm{Na}_{2} \mathrm{HPO}_{4}, 1 \mathrm{NaHCO}_{3}, 20$ glucose, $10 \mathrm{Na}$-HEPES, $\mathrm{pH}$ 7.3-7.4 (osmolarity, $315 \mathrm{mOsm}$ ). In most experiments (as indicated), CNQX (1-3 $\mu \mathrm{M})$, a non-NMDAR antagonist, was added to lower AMPA peak current amplitude and reduce voltage-clamp errors. Pipette solution contained (in mM): $112.5 \mathrm{~K}$-methansulfonate, $8 \mathrm{NaCl}, 5 \mathrm{MgATP}, 20$ HEPES, 0.2 BAPTA, $20 \mathrm{~K}_{2}$-creatine phosphate, $50 \mathrm{U} / \mathrm{ml}$ creatine phosphokinase, $\mathrm{pH}$ 7.2. Neurons were clamped at $-65 \mathrm{mV}$ with Axopatch 200B. Currents were digitized at $5 \mathrm{kHz}$ and low-pass filtered at $1 \mathrm{kHz}$. The series resistance was compensated $60-70 \%$. Only recordings with series resistance $<20 \mathrm{M} \Omega$ were analyzed. Autaptic EPSCs were evoked by depolarizing the cells from -65 to $0 \mathrm{mV}$ for $3 \mathrm{msec}$. Holding potentials were not corrected for the liquid junction potential. All experiments were performed at room temperature $\left(22-25^{\circ} \mathrm{C}\right)$.

To monitor NMDAR-mediated EPSC (NMDAR EPSC) and the progressive blockade of NMDAR EPSC by (+)-5-methyl-10,11-dihydro-5Hdibenzo [a,d]cyclohepten-5,10-imine maleate (MK-801), computercontrolled solenoid-driven valves were used to rapidly switch between two gravity-fed extracellular solutions without and with $5 \mu \mathrm{M}$ MK-801 in which $10 \mu \mathrm{m}$ glycine and $5 \mu \mathrm{M}$ CNQX were added, and the $\mathrm{MgCl}_{2}$ concentration was reduced from $1 \mathrm{~mm}$ to nominal $0 \mathrm{~mm}$. The solution was applied via a multibarrel manifold tip (250 $\mu \mathrm{m}$ tip) (AutoMate Scientific) positioned $\sim 1$ $\mathrm{mm}$ from a recording cell.

To address the extent of MK-801 washout, we used low-frequency stimulation $(0.1 \mathrm{~Hz})$ to monitor NMDAR EPSCs after ceasing to perfuse MK-801 and observed no significant difference in the ratio of the first to second NMDAR EPSC amplitude for cases with or without MK-801 applied previously during train stimulation $(p>0.05)$. This means that the interval (1-2 min) between the end of the train (train/MK-801) paradigm (see Fig. $5 A$ ) and the beginning of the NMDAR EPSC monitoring period was enough time to wash out MK-801. If MK-801 had not been washed out, we would have expected the second EPSC to be significantly smaller because it would be subject to continued MK-801 block.

Imaging. Imaging of neuronal $\mathrm{Ca}^{2+}$ transients was performed with wide-field microscopy using a Zeiss Axioskop FS microscope (Zeiss, Oberkochen, Germany) equipped with an intensified CCD camera (Stanford Photonics) as described previously (Mackenzie et al., 1996), using a $60 \times$ water immersion 0.9 numerical aperture objective (Olympus, Tokyo, Japan) for optical recordings. The camera acquired data at 15 frames per second (pixel size $=0.2 \mu \mathrm{m}$ ), and the images were captured to a PC using a frame grabber (EPIX, Buffalo Grove, IL). BAPTA in the pipette solution was substituted with Fluo-4 $(0.4 \mathrm{~mm})$ and mag-fura-2 (0.2-0.3 mM) for $\mathrm{Ca}^{2+}$ imaging. Mag-fura-2, a low-affinity $\mathrm{Ca}^{2+}$ indicator with high fluorescence at basal $\left[\mathrm{Ca}^{2+}\right]_{\mathrm{i}}$ at $380 \mathrm{~nm}$ excitation (Raju et al., 1989) was used to resolve the fine processes under resting conditions, and Fluo- 4 was used to view local changes in $\left[\mathrm{Ca}^{2+}\right]_{\mathrm{i}}$. For each optical recording trial, we collected 20 sec of Fluo- 4 images (300 frames; at $490 \mathrm{~nm}$ excitation) and then switched to $380 \mathrm{~nm}$ excitation to collect one averaged ( $1 \mathrm{sec}$ ) mag-fura-2 image for normalization (see below). The excitation light was delivered to the microscope using a flexible fiber optic cable coupled to a filter wheel and an HBO $100 \mathrm{~W}$ arc lamp. On the emission side we used a Fura/Fluo-4 dichroic mirror (Chromo Technologies, catalog \#74000) combined with a 540/40 nm emission filter.

For electrophysiological experiments in which relative changes in AMPA receptor-mediated miniature EPSCs (AMPAR mEPSCs) were measured, $0.2 \mathrm{~mm}$ BAPTA was included in the pipette solution, and simultaneous $\mathrm{Ca}^{2+}$ imaging was not performed (in most experiments). In initial experiments we found that BAPTA afforded better recording conditions than the Fluo-4 and allowed us to collect more trials evaluating mEPSC rate after train stimulation. Because BAPTA has a twofold lower $K_{\mathrm{d}}$ (160 vs $345 \mathrm{~nm}$ ) than Fluo-4 (Molecular Probes, Eugene, OR; data table), we used $0.2 \mathrm{~mm}$ BAPTA for electrophysiology-only experiments and $0.4 \mathrm{~mm}$ Fluo- 4 for imaging to match buffer capacity under the two experimental conditions (buffer concentration $/ K_{\mathrm{d}}$ buffer $\sim$ buffer capacity; 1250 BAPTA vs 1160 Fluo-4). Buffer capacity (a measure of bound $\mathrm{Ca}^{2+}$ to free $\mathrm{Ca}^{2+}$ at equilibrium) and the $\mathrm{Ca}^{2+}$ extrusion rate are the primary determinants of $\mathrm{Ca}^{2+}$ clearance (Neher and Augustine, 1992; Helmchen and Tank, 2000). To check that the use of BAPTA $(0.2$ $\mathrm{mm})$ versus Fluo-4 (0.4 mM) did not affect the relative time course of asynchronous release, we recorded AMPAR-mediated asynchronous mEPSCs in three neurons in which imaging experiments were also performed in the presence of Fluo-4 (0.4 mM). We then calculated relative changes in whole-cell current variance during the asynchronous phase of release to provide a relative measure of mEPSC rate decay (as done for electrophysiological experiments; see Fig. 4). Fitting the fast component of the decay of variance, we observed only a modest difference between cells loaded with Fluo-4 (0.4 mM) or BAPTA (0.2 mM): $257 \pm 16$ and $213 \pm 16 \mathrm{msec}$, respectively. This analysis confirms that our electrophysiological conditions are comparable with those under which imaging experiments were performed. Importantly, in all imaging trials we also recorded the time course of asynchronous NMDAR-dependent synaptic activity and found that it was well correlated with AMPA mEPSC frequency determined from electrophysiological experiments (at periods $0.8-18$ sec after train stimulation).

In the extracellular solution, $1 \mathrm{~mm}$ D- $\alpha$-amino adipate (D-AA), a lowaffinity competitive NMDAR antagonist, was added to block NMDAR activation during train stimulation and to permit rapid NMDAR unblocking after washout. To monitor $\mathrm{Ca}^{2+}$ signals through NMDARs, the extracellular solution without NMDAR antagonists $\left(\mathrm{MgCl}_{2}\right.$ and D-AA free) was applied using computer-controlled solenoid-driven valves via a glass pipette $(\sim 200 \mu \mathrm{m}$ tip, inner diameter $)$ positioned $\sim 400 \mu \mathrm{m}$ from a recording cell. Imaging trials were captured at $>1$ min intervals to reduce synaptic depression and potential toxic effects of NMDAR activation.

Fluo-4 and mag-fura- 2 were purchased from Molecular Probes. D,LAPV was purchased from Precision Biochemicals (Vancouver, British Columbia, Canada). D-AA and other chemicals were purchased from Sigma (St. Louis, MO).

Data analysis. Phasic and asynchronous components of release as well as RRP size during $20 \mathrm{~Hz}$ stimulation for $0.5 \mathrm{sec}$ were measured as described previously (Otsu et al., 2004). Currents were analyzed off-line using programs written in the Interactive Data Language (IDL) (Research System, Boulder, CO) programming language on a Pentium processor-based computer. Spontaneous events (mEPSC) were detected 
with an event detection program that is a built-in component of AxoGraph 4.0 (Axon Instruments, Foster City, CA). A template of event was set: $1.5 \mathrm{msec}$ rise time, $3 \mathrm{msec}$ decay time. An event peak was detected with a criteria of a threshold $>3 \times$ SD of the baseline noise. In MK-801 experiments, a decay time constant of NMDAR EPSC was measured with a monoexponential fitting at $20-400 \mathrm{msec}$ after stimulus onset. The decay constants for use-dependent block of NMDAR EPSCs by MK-801 were measured by fitting to the following function: $y=f_{1} \exp \left(-\mathrm{x} / t_{1}\right)+f_{2}$ $\exp \left(-\mathrm{x} / t_{2}\right)+C$, allowing $f_{1}, f_{2}, t_{1}, t_{2}$ and $C$ to vary. A weighted time constant was calculated by $\left(f_{1} t_{1}+f_{2} t_{2}\right) /\left(f_{1}+f_{2}\right)$.

To identify dendritic regions showing MSCTs, we constructed difference images by first averaging fluorescence over $1 \mathrm{sec}$ periods (15 images). Images of change in fluorescence (difference images) were then produced by subtracting consecutive averaged images (image pairs) and determining their absolute value. The difference images were then averaged, and a single image was created that reflected sites that exhibited the greatest changes in fluorescence over the $10 \mathrm{sec}$ sampling epoch. These potential sites of MSCTs were further evaluated using plots (pixel value vs time) from $\sim 2-3 \mu \mathrm{m}^{2}$ areas of interests $(7 \times 7$ or $9 \times 9$ pixels). The change in Fluo-4 fluorescence intensity $\left(F_{490}\right)$ of each area of interest was divided by the mag-fura- 2 fluorescence $\left(F_{380}\right)$ to adjust for differences in dye filling and focus along the dendritic tree $\left(F_{490} / F_{380}\right)$.

The sites of MSCT origin were defined as described previously (Murphy et al., 1995) by monitoring the rising phase of the $\mathrm{Ca}^{2+}$ transient and selecting the dendritic region with the earliest rise. The initiation time of the $\mathrm{Ca}^{2+}$ transient was defined as the first point of three consecutive measurements ( 66 msec interval) that was $>2$ SDs above the mean baseline fluorescence. The baseline fluorescence was calculated by averaging at least three points before the initiation of an MSCT. The amplitude of MSCTs was measured by selecting the maximum fluorescence value within five consecutive points after the MSCT initiation point. Usually the fluorescence peaked within the first three data points. In some cases slow transient elevations in $\left[\mathrm{Ca}^{2+}\right]_{\mathrm{i}}$ were observed $(>500 \mathrm{msec}$ rise time); these types of events were not considered MSCTs. Imaging analysis was performed using custom routines written in the IDL. Line-plot analysis in Figure $3 B$ was done using the Multiple Kymograph plug-in for ImageJ written by Jens Rietdorf (http://rsb.info.nih.gov/ij/). Using this plug-in, multiple line plots are created from the average pixel value across an irregular line of seven to nine pixel widths (placed along the dendrite) and stacked to generate a "line scan" type image, in which the $X$ dimension corresponds to line length and the $Y$ dimension corresponds to time. For presentation the line-scan image was smoothed with a $3 \times 3$ filter. Statistical analysis was done by Prism version .3.0 (GraphPad Software, San Diego, CA). Origin 6.1 (Microcol Software, Northampton, MA) was used for curve fitting. Data are displayed as mean \pm SEM.

\section{Results}

A switch to asynchronous release during trains of stimulation We have used autaptic microisland cultures to directly measure the rate of glutamate release and its postsynaptic action at individual synapses after trains of stimulation. In $3 \mathrm{~mm}$ extracellular $\mathrm{Ca}^{2+} / 1 \mathrm{mM} \mathrm{Mg}^{2+}$, neurons showed robust depression (>90\%) of evoked synaptic responses after only $0.5 \mathrm{sec}$ of $20 \mathrm{~Hz}$ stimulation (Fig. 1A). After trains of evoked synaptic stimuli, a large inward current and decaying tail were observed that were composed of asynchronously released quanta. These developing neurons in autaptic culture (4-10 d in vitro) undergo robust asynchronous release without the requirement to use $\mathrm{Sr}^{2+}$ or other agents to prolong time course of release (Abdul-Ghani et al., 1996). Previous studies as well as our own work indicate that asynchronous release is dependent on elevated intraterminal residual $\mathrm{Ca}^{2+}$ concentration because it is selectively blocked by treatment with the relatively slow chelator EGTA-AM (Cummings et al., 1996; Atluri and Regehr, 1998; Otsu et al., 2004). Given that the individual AMPAR mEPSCs making up the tail have relatively rapid decay kinetics $(<5 \mathrm{msec})$, the decay of the tail current reflects the time course of asynchronous release fre-
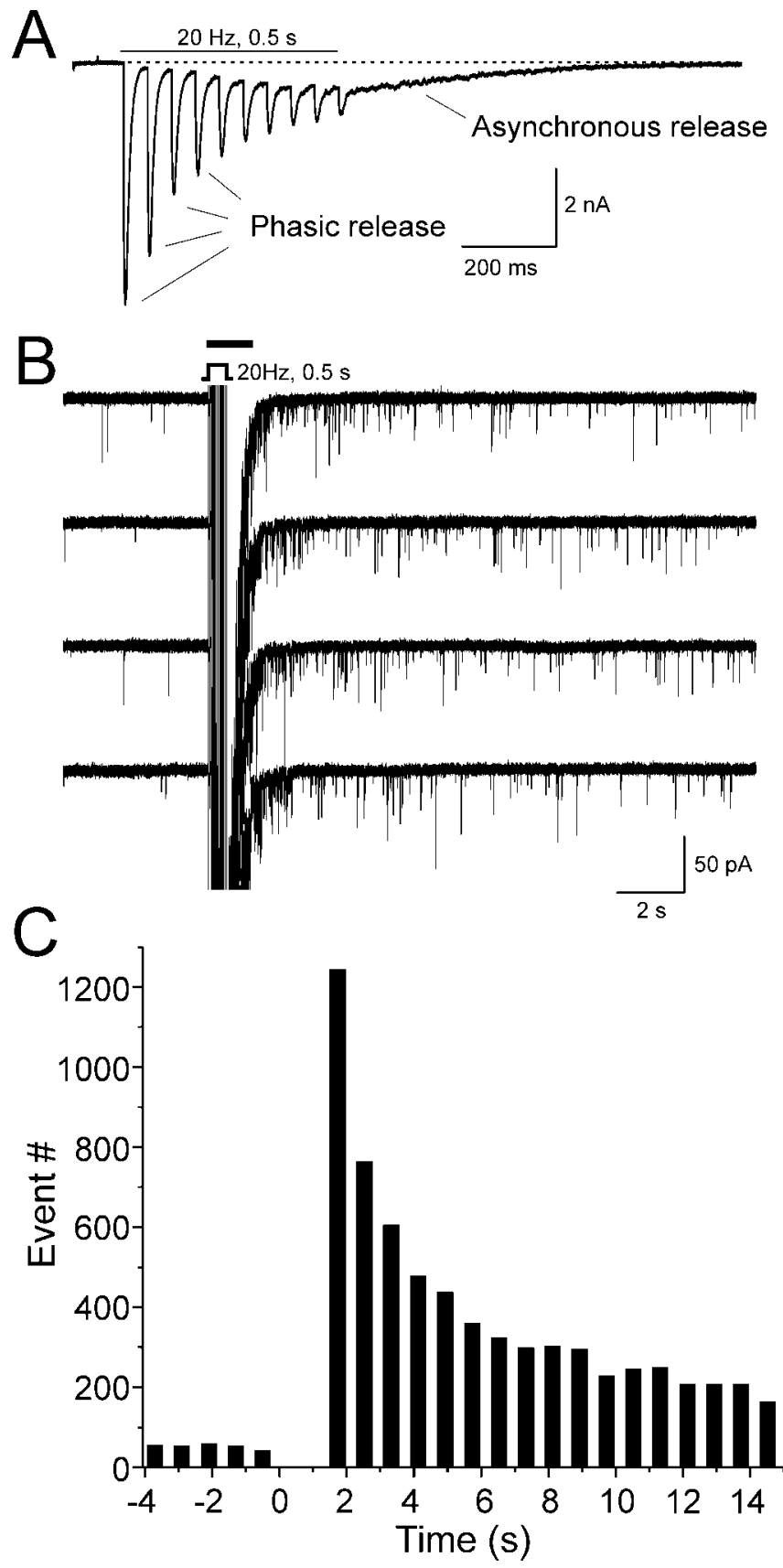

Figure 1. Asynchronous release continues well after phasic release depression. $A$, Averaged whole-cell current $(n=3)$ induced by train stimulation ( $20 \mathrm{~Hz}$, 10 pulses). B, Delayed mEPSCS after train stimulation in four consecutive trials in the same cell in $A$ are shown (time scale compressed compared with A). C, mEPS( event histogram plotted for 70 total trials in six cells. Bin size is $0.8 \mathrm{sec}$. Time 0 indicates the end of train stimulation. We did not count $m$ EPSC events within 1.33 sec after train stimulation because individual events could not be unambiguously detected at this time. These experiments were done under conditions (100 $\mu \mathrm{m} \mathrm{D,L-APV}$ and 3 $\mathrm{mm} \mathrm{CaCl}$ and $\left.1 \mathrm{~mm} \mathrm{MgCl}{ }_{2}\right)$ to maximize AMPAR mEPSCs, which are easier to detect than NMDAR $\mathrm{mEPSC}$ because of their greater amplitude and faster kinetics.

quency. We assume that the tail current is derived primarily from the summation of individual quanta with decay constants much shorter than the time course of asynchronous release (Otsu et al., 2004). The mean amplitude of the tail current correlated linearly with its variance over the first $1.5 \mathrm{sec}$ after train simulation (Otsu et al., 2004), indicating that the tail current was composed of individual events that have a similar mean amplitude over time. If the tail current is made up of linearly additive elementary com- 
ponents that occur at random intervals, the amplitude of the elementary components is equal to $2 \times$ (variance)/(mean) (Katz and Miledi, 1972). Therefore, on a plot of mean versus variance the slope should be constant and a measure of quantal amplitude. Although the analysis of mean and variance can be used to derive relative changes in $\mathrm{mEPSC}$ rates just after train stimulation (0.05-1.5 sec) (Otsu et al., 2004), we observed enhanced mEPSC rates well after train stimulation that continued for up to $15 \mathrm{sec}$ (Fig. $1 B)$ ( $y$-axis greatly expanded to emphasize the mEPSCs). At periods $>1.5 \mathrm{sec}$ after the train, mEPSC rates were generally too low to be accurately measured from the mean amplitude of the current (and to a lesser extent, variance after $6 \mathrm{sec}$ ) because baseline offsets could be significant compared with the amplitude contributed by mEPSCs. At these later times we used a semiautomated mEPSC analysis routine that identified individual mEPSCs by their amplitude, rise, and decay times (see Materials and Methods). This analysis revealed that elevated mEPSC rates persisted for even up to $15 \mathrm{sec}$ after a train (decay time constant $9.1 \pm$ $4.3 \mathrm{sec}$ for slow component; 11\% amplitude) (Fig. 1C). Because there was overlap in the mEPSC rate time courses (derived from the different methods, variance, and semiautomated direct detection) (see Fig. 4), we were able to append the time courses measured using different approaches to produce a full description of the mEPSC rate decay after train stimulation (see Fig. 4). Our approach to assessing changes in release frequency over time was to use current variance for the first $6 \mathrm{sec}$ and semiautomated direct AMPAR mEPSC detection for later times when rates were relatively low and superposition of events was minimal. As described previously, we estimated the peak rate of mEPSCs in units of RRPs per second (Otsu et al., 2004). In this case the RRP was defined as the cumulative postsynaptic response attributed to a high-frequency stimulation without a constant resupply of releasable quanta. With a $0.5 \mathrm{sec}$ train delivered at $20 \mathrm{~Hz}$ (the stimulus used for subsequent imaging experiments), we estimate that neurons release $2.1 \pm 0.2$ RRPs per second of transmitter at peak ( $n=5$ cells). By using the electrophysiological approaches described above, we can get an accurate description of relative changes in mEPSC rates after train stimulation within a population of synapses; however, these methods do not permit determination of the actual quantal release rates at individual synapses.

\section{Imaging of asynchronous postsynaptic activity after train stimulation}

By using individual hippocampal neurons with autaptic synapses in microisland culture, we monitored the postsynaptic rate of asynchronous activity at individual dendritic sites after train stimulation by using fluorescent $\mathrm{Ca}^{2+}$ indicators to image NMDAR mEPSCs as described previously (Umemiya et al., 1999, 2001; Murthy et al., 2000). An advantage of autaptic synapses is that all release events are mediated by a single axon, and therefore synaptic depression is not mediated by selective loss of impulse conduction between axons. A complication of using the autaptic microisland cultures, however, is that the action potentials (APs) that trigger synaptic release propagate to postsynaptic compartments leading to $\mathrm{Ca}^{2+}$ signals attributed to both the AP and the postsynaptic effect of the transmitter. Because the signal resulting from the AP should be relatively constant throughout trials, we subtracted it to resolve signals attributed to individual quanta that were released asynchronously. To differentiate between $\mathrm{Ca}^{2+}$ signals mediated by phasic and asynchronous release, we applied the rapidly dissociating NMDAR antagonist D-AA (1 $\mathrm{mM}$ ) before and during the 10 stimuli delivered at $20 \mathrm{~Hz}$ to produce phasic release (in the presence of $1 \mathrm{mM} \mathrm{Mg}^{2+}$ ); then the
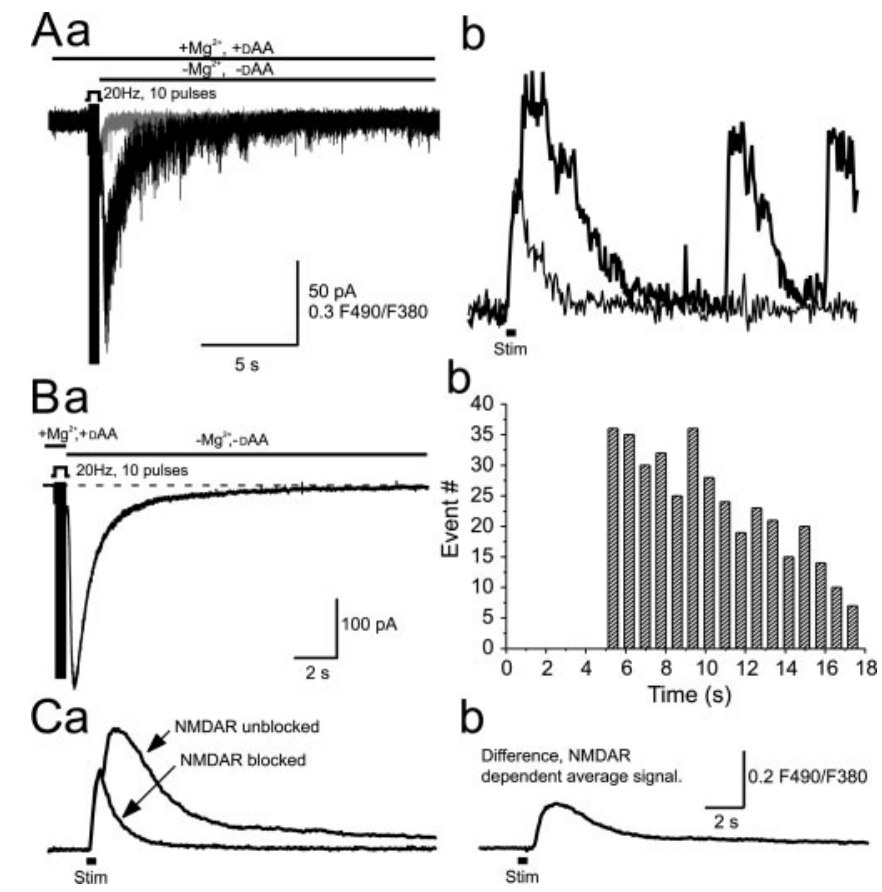

Figure 2. $\mathrm{Ca}^{2+}$ transients mediated by NMDA receptors induced by asynchronous release. $A a$, Two whole-cell current traces are superimposed. One is recorded with $\mathrm{Mg}^{2+}$ and D-AA (1 $\mathrm{mm}$ ) present (NMDARs were blocked) (gray line). Just after train stimulation, the second trace was made by washing out the $\mathrm{Mg}^{2+}$ - and D-AA-containing extracellular solution after the last pulse of train (10 stimuli at $20 \mathrm{~Hz}$ ) (black line). CNQX (1-3 $\mu \mathrm{M}$ ) was included in the solution to reduce AMPAR activity. $A b$, Time course of two superimposed $\mathrm{Ca}^{2+}$ records (ratio of Fluo- 4 to mag-fura-2 fluorescence, $F_{490} / F_{380}$ ) that were obtained under the two different conditions [NMDAR blocked (thin line) and unblocked (thick line) during asynchronous release period] for the cell described in $A a$. MSCTs were clearly observed after train stimulation under conditions that unblock NMDARs. Ba, Time course of the average NMDAR-induced current associated with asynchronous release, obtained under $\mathrm{Mg}^{2+}$ - and D-AA-free solution after train stimulus ( $n=$ 5 cells). $B b$, Frequency histogram for MSCTs recorded under $\mathrm{Mg}^{2+}$ - and D-AA-free solutions after train stimulation (data shown is from 80 synaptic sites in 5 cells and includes 375 events). The zero point is at the onset of the train stimulus. Data from 0 to 5 sec was not analyzed because of event overlap and $\mathrm{Ca}^{2+}$ indicator saturation (see Results and Discussion). (a, Average fluorescence change (in units of $\left.F_{490} / F_{380}\right)$ associated with train stimulation under conditions in which NMDARs are blocked after train stimulation or unblocked by washout of $\mathrm{Mg}^{2+}$ and D-AA (average of 29 sites and 8 trials). $C b$, Plot of the difference between the records in Ca indicating the NMDAR-dependent $\mathrm{Ca}^{2+}$ signal associated with asynchronous release.

NMDAR antagonist was rapidly washed out (over $\sim 400 \mathrm{msec}$ ), and the solution was replaced by one lacking $\mathrm{Mg}^{2+}$ to efficiently view the NMDAR-mediated postsynaptic $\mathrm{Ca}^{2+}$ component of asynchronously released quanta (Fig. 2A) [compare videos 1 and 2 (supplemental material, available at www.jneurosci.org), taken under conditions that block and unblock, respectively, NMDARs after train stimulation (site shown in Fig. 3A).] Under these conditions we expected that $\mathrm{Ca}^{2+}$ signals were caused by both the residual effects of the APs and asynchronous transmitter release. $\mathrm{Ca}^{2+}$ transients from control trials in which NMDAR antagonists were present during and for $17 \mathrm{sec}$ after train stimulation were subtracted from all records to isolate an NMDAR-mediated optical signal attributed to asynchronous release (Fig. 2Aa,C). For an example of subtracted images demonstrating only NMDAR-dependent asynchronous release, see video 3 (supplemental material, available at www.jneurosci.org). In video 3 (supplemental material, available at www.jneurosci.org), very robust asynchronous release-mediated $\mathrm{Ca}^{2+}$ changes are observed within the first $3 \mathrm{sec}$ after train stimulation; after $\sim 5 \mathrm{sec}$, individual MSCT (bright flashes) events can be readily observed at par- 
ticular sites. In the presence of NMDAR antagonist we expect $\mathrm{Ca}^{2+}$ signals to arise from the slow decay of AP-associated $\mathrm{Ca}^{2+}$ entry (video 1, available at www.jneurosci.org as supplemental material). For example, in Figure $2 A b$, a record is shown that depicts a $\mathrm{Ca}^{2+}$ transient produced in the presence of $\mathrm{Mg}^{2+}$ and D-AA both during train stimulation and over a $17 \mathrm{sec}$ period that follows. A robust increase in intracellular $\mathrm{Ca}^{2+}$ followed by an exponential decay was observed and attributed to AP-mediated opening of voltage-gated $\mathrm{Ca}^{2+}$ channels alone (Fig. $2 \mathrm{Ab}$, thin line). If the stimulus was repeated and the NMDAR antagonists were washed out just after the train, we observed that the $\mathrm{Ca}^{2+}$ transient now decayed in a more complex manner (Fig. $2 \mathrm{Ab}$, thick line). Examination of records from individual sites revealed MSCT-like peaks that were attributed to individual quantal transmitter release events. Subtraction of the two traces (NMDAR unblocked minus NMDAR blocked) revealed the NMDAR antagonist-sensitive, late asynchronous release component (Figs. 2C, $3 A$ ). In the same cells we also recorded whole-cell currents under conditions expected to isolate NMDAR activity and observed a slow average current apparently composed of many NMDAR mEPSCs that decay with a time course similar to the elevated rate of MSCTs observed (Fig. 2B). In five cells we plotted the appearance of these MSCT events 5-17 sec after train stimulation and observed a fall in rate with a time constant similar to that for the slow component of the NMDAR current, suggesting that these events were induced by the train stimulus (Fig. $2 \mathrm{Bb}$ ). By averaging fluorescence signals from 8 trials and 29 sites, we were able to establish that washout of D-AA and $\mathrm{Mg}^{2+}$ (after a train stimulation) effectively reveals NMDAR-mediated changes in $\left[\mathrm{Ca}^{2+}\right]_{\mathrm{i}}$ attributed to elevated MSCT frequency (Fig. $2 \mathrm{C}$ ). The average $\mathrm{Ca}^{2+}$ transient decays with a time course similar to that for the NMDAR current activated by asynchronous activity; however, one must consider that individual MSCTs have longer kinetics than the NMDAR mEPSCs.

Evidence that the late $\mathrm{Ca}^{2+}$ transients were MSCTs included their complete suppression in solutions with NMDAR blockers (Fig. 2Ab) (compare videos 1 and 2, available at www.jneurosci.org as supplemental material) and a close correspondence between the amplitude and time course of spontaneous MSCTs and those evoked asynchronously by train stimulation in the same cell (Fig. 3A). The asynchronous release-dependent $\mathrm{Ca}^{2+}$ transients have considerably slower decay kinetics than NMDAR mEPSCs reported previously (Umemiya et al., 2001). This is expected because the $\mathrm{Ca}^{2+}$ transient is slowed (with respect to the current that triggers it) by relatively slow intracellular extrusion mechanisms and both endogenous and exogenous $\left(\mathrm{Ca}^{2+}\right.$ indicator) buffering (Sabatini et al., 2002). To measure asynchronous $\mathrm{Ca}^{2+}$ transients, we found it necessary to use a relatively high concentration of a high-affinity $\mathrm{Ca}^{2+}$ indicator (0.4 mM Fluo-4). The use of this indicator led to some saturation associated with the AP train and initial, very robust asynchronous release (Fig. $2 A b$, thick unsubtracted record). Because of saturation we did not use the MSCT data for periods $<5 \mathrm{sec}$ after train stimulation for estimation of peak MSCT rates. In addition, MSCTs decay relatively slowly ( $\sim 2 \mathrm{sec}$ decay constant), and it was possible to have overlapping events that would lead to an underestimate of MSCT frequency. Because we use a high-affinity dye, MSCT amplitudes may be affected by saturation, and thus we used the $\mathrm{Ca}^{2+}$ indicators to measure relative changes in event frequency and not amplitude. Lower-affinity indicators would solve many of these problems at the expense of considerably smaller fluorescence changes, raising the possibility of missed events. Although unlikely (Mainen et al., 1999), it is also possible that NMDAR-

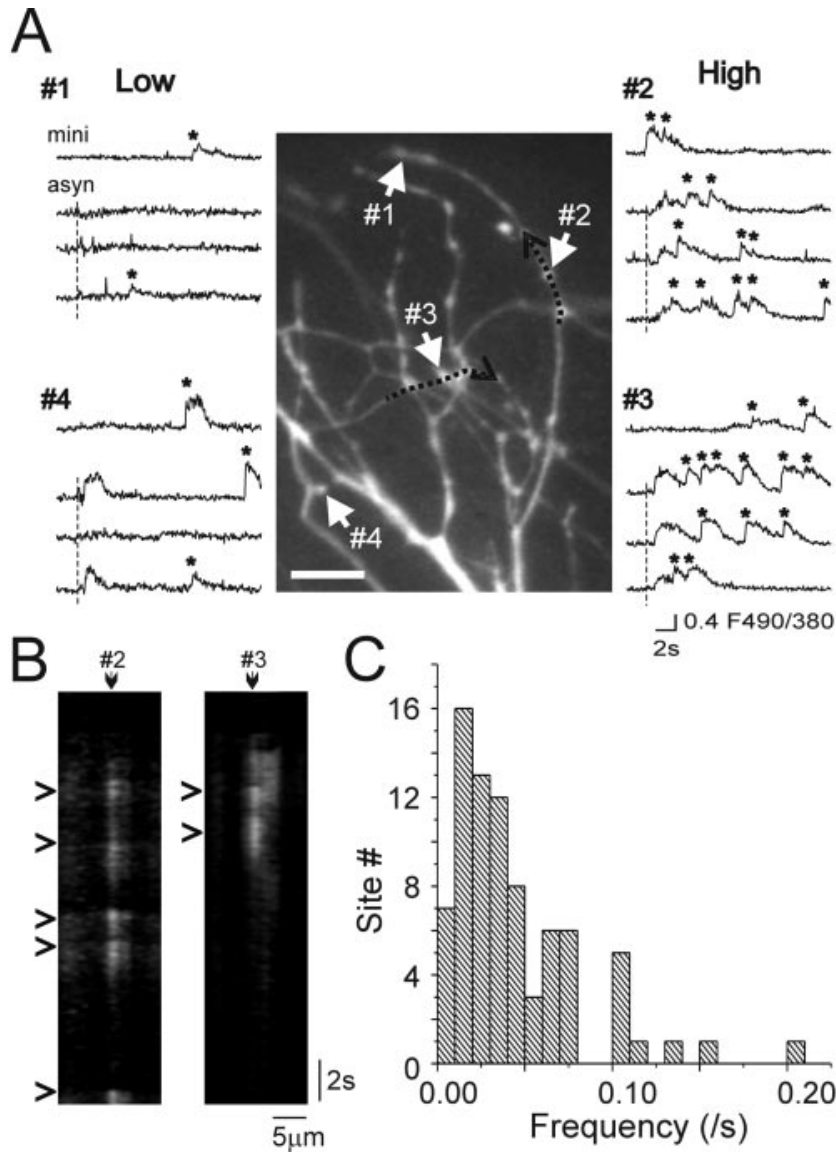

Figure 3. Calcium transients mediated by asynchronous release at individual synapses. $A$, The records shown are plots of $\mathrm{Ca}^{2+}$ responses against time at four dendritic sites [ratios of Fluo-4 fluorescence divided by mag-fura-2 fluorescence, a low-affinity $\mathrm{Ca}^{2+}$ probe used to measure dendrite structure (see Materials and Methods)]. Examples of synapses showing a low (left column) and high (right column) frequency of MSCT activity after train stimulation. Four traces are shown from each site indicated in the center column. MSCTs were initiated and measured at $\sim 2 \mu \mathrm{m}^{2}$ regions centered over the indicated sites. The top trace is taken without stimulation and reflects spontaneous miniature synaptic activity. The three bottom traces reflect train-induced asynchronous MSCTs that are NMDAR dependent (subtracted signals; ratio recorded under $\mathrm{Mg}^{2+}$ - and $\mathrm{D}$-AA-free condition minus $\mathrm{Mg}^{2+}$ - and $\mathrm{D}-\mathrm{AA}$-containing conditions). Detected events are indicated by an asterisk. Vertical dashed lines indicate the point just before train stimulation $(20 \mathrm{~Hz}, 0.5 \mathrm{sec})$. Center column, Basal mag-fura- 2 image of a dendritic region where $\mathrm{Ca}^{2+}$ dynamics were measured. The broken lines were analyzed in a line-plotting mode and span sites 2 and 3 as described below. Scale bar, $10 \mu \mathrm{m}$. $B$, Line-plot analysis of repeated MSCT initiation at sites 2 and 3 indicated in $A$ (fourth sweep shown), made by averaging across the width of a 7- to 9-pixel-thick line placed over the dendritic regions and in some case extracellular space as indicated (15.6 $\mu \mathrm{m}$ total line length; data shown is raw Fluo-4 fluorescence). The $X$ dimension of the image corresponds to line length and the $Y$ dimension corresponds to time (image composed of stacked lines derived from each frame). At site 2, four repeated MSCTs were observed within a segment that was $<2 \mu \mathrm{m}$. At site 3 , the first change in fluorescence was ambiguous and may have been associated with signal spread from an adjacent site and was not counted; a few seconds later, two MSCTs were observed (indicated by arrowheads) centered within the same dendritic segment. C, Frequency histogram for traininduced MSCTs from each site (80 sites from 5 cells); only MSCTs 5 sec after train stimulation were included.

mediated release of $\mathrm{Ca}^{2+}$ from stores may increase the amplitude of the events that we observe, providing another reason for monitoring only event frequency and not amplitude (Emptage et al., 2003).

Although we are being conservative in only monitoring MSCT event frequency and not amplitude, $\mathrm{Ca}^{2+}$ or fluorescence signal spill-over between adjacent synapses may lead to inaccuracy in asynchronous $\mathrm{Ca}^{2+}$ transient frequency determination. To re- 
duce the likelihood of signal spillover, we have avoided analyzing sections of dendrites in which multiple processes overlap. Furthermore, synaptic density in cultured cells is considerably lower than that found in vivo, reducing the likelihood of signal spillover. In previous studies using dissociated cultures, we reconstructed sites of MSCTs and found that most $\mathrm{Ca}^{2+}$ transient initiation sites were associated with a single presynaptic terminal (Mackenzie et al., 2000); however, it is conceivable that some differences in $\mathrm{Ca}^{2+}$ transient frequency may be caused by differences in synapse or release site number. Line-plot analysis of $\mathrm{Ca}^{2+}$-induced changes in fluorescence during repeated MSCTs indicated little spatial variation (within the limits of light microscopy and our imaging conditions) with regard to $\mathrm{Ca}^{2+}$ transient initiation points during multiple events at presumably the same synapse (Fig. 3B). Arguing against significant overlap in MSCT initiation sites (leading to higher apparent event frequency) is the relatively small positive skew in the distribution of MSCT frequencies (Fig. 3C). Positive skew could arise through the relatively infrequent overlap of individual synaptic MSCT initiation sites, creating relatively low-incidence sites of relatively higher frequency. If sites with higher MSCT rates were caused by overlapping synapses, the distribution suggests that they are a minority ( $\sim 10 \%$ of the sites), with no more than two- to threefold the rate of the MSCTs observed in the general population presumably attributed to one synapse.

\section{Estimates of peak release rates from asynchronous miniature synaptic calcium transients}

To evaluate how the frequency of MSCTs at individual synapses corresponds to the decay in asynchronous release rates, we examined the temporal distribution of MSCT events after 10 synaptic stimuli given at $20 \mathrm{~Hz}$ by plotting the times at which MSCT events were initiated on the histogram shown (Fig. $2 B b)(0.8 \mathrm{sec}$ bins). As mentioned above, $\mathrm{Ca}^{2+}$ concentration in dendrites was very high initially just after train stimulation for several reasons, including the activation of voltage-gated $\mathrm{Ca}^{2+}$ channels in dendrites (associated with backpropagating APs) and a robust amount of asynchronous release. After $\sim 5 \mathrm{sec}$, however, individual MSCTs could be reliably detected. During this time period ( $>5 \mathrm{sec}$ to end of record), the relative decay kinetics for MSCT frequency matched that of mEPSC frequency (time course derived from analysis of AMPAR mEPSCs) (Fig. 4A,B). Our assumption was that the relative frequency of MSCT appearance (event initiation times) during this period should reflect the underlying mEPSC frequency. Therefore, by knowing the decay of MSCT frequency over a time period well after train stimulation and assuming a similar decay time course for appearance of AMPAR mEPSCs, we estimated what the frequency of MSCTs would have been at time $=0$ (by extrapolation to the AMPAR mEPSC rates). Because MSCT frequency can be expressed in units of events per synapse per second, we could get an estimate of the peak rates of quantal release for times when event frequency was too high for direct measurement by $\mathrm{Ca}^{2+}$ imaging. For the synapses shown in the graph in Figure 3C, we observed an average rate of MSCTs of $0.045 \pm 0.004$ events per synapse per second ( 80 sites) over a period ranging from 5 to $17 \mathrm{sec}$ after train stimulation. To determine how well the decay of MSCT rates agreed with whole-cell-derived mEPSC rates, we plotted the average MSCT rate per synapse alongside relative changes in AMPAR mEPSC frequency measured by variance or direct event detection (Fig. $4 A, B)$. To align the MSCT frequency plots with these other measures of activity, we multiplied the AMPAR mEPSC current variance, the NMDAR average current (see below), and the decay in
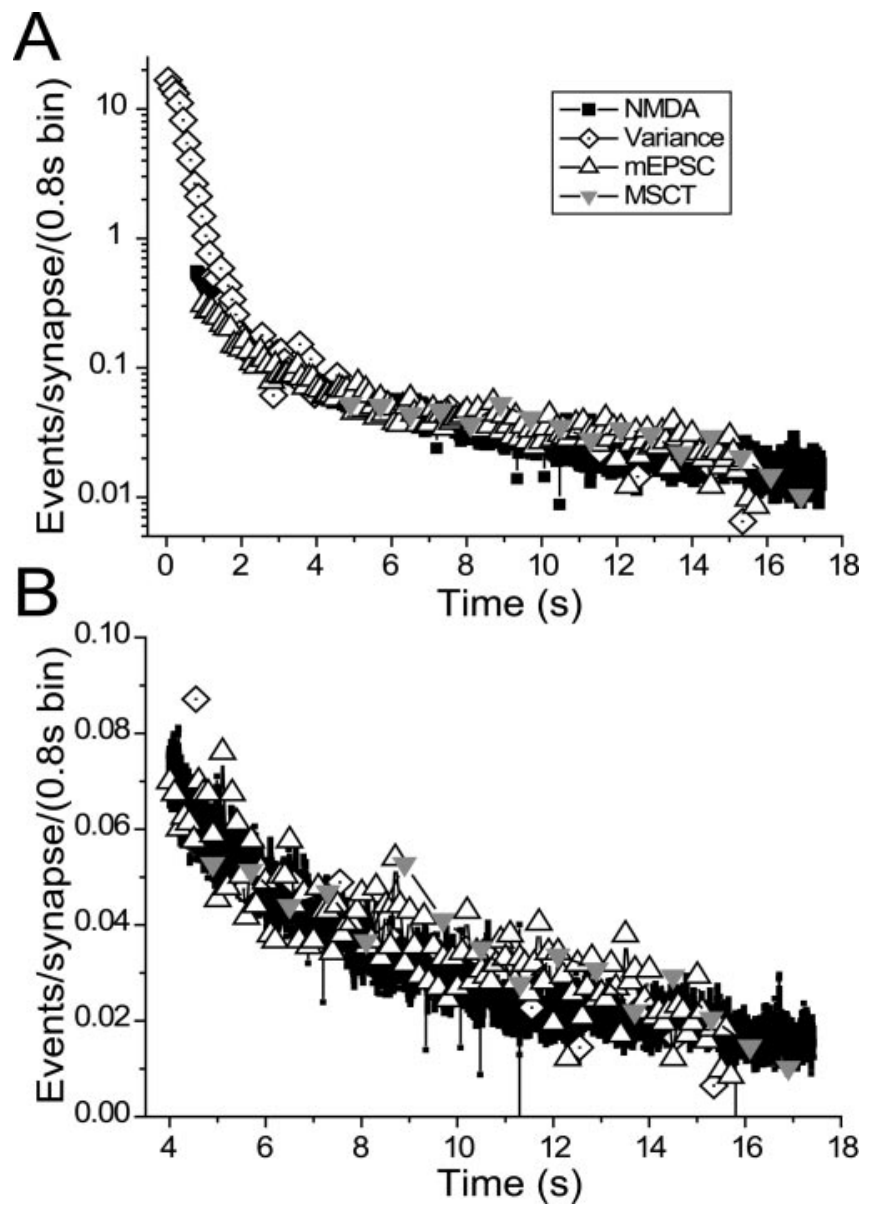

Figure 4. Estimating the peak rate of asynchronous release at individual synapses. A, Plots of various measures of asynchronous release were made over the indicated times after $20 \mathrm{~Hz}(0.5$ $\mathrm{sec}$ ) train stimulation. MSCT rates are expressed in units of events per synapse per $0.8 \mathrm{sec}$ bin ( 375 events from 5 cells) (inverted triangle). Because MSCTs have a prolonged time course, we only plotted the time of their appearance and not their full time course. Given $\mathrm{Ca}^{2+}$ indicator saturation and event overlap, MSCT appearance was only plotted for times $>5$ sec after train stimulation. AMPA mEPSC frequency were measured from $1.05 \mathrm{sec}$ after train stimulation and plotted every 100 msec (7634 events, 70 trials from 6 cells) (triangle). The average NMDAR current under NMDAR-unblocked conditions after train stimulation (18 traces from 5 cells) (as described in Fig. $2 \mathrm{Aa}$ ) was plotted from $0.8 \mathrm{sec}$ after train stimulation (square). This period ( 0.8 $\mathrm{sec}$ ) is necessary to wash out $\mathrm{Mg}^{2+}$ and D-AA to activate NMDARs. Average variance of synaptic currents after train stimulation (39 traces from 6 cells) was plotted, measured over $100 \mathrm{msec}$ bins, and reflects subtraction of variance of currents before train stimulation (diamond); for time points $>3.95 \mathrm{sec}$, the variance was averaged over longer intervals ( $0.6 \mathrm{sec}$ interval from 3.95 to 4.55 sec and 1 sec intervals thereafter). The different measures of asynchronous synaptic activity decay, AMPAR mEPSC frequency, NMDAR currents, or AMPAR mEPSC current variance were adjusted by multiplying by an amplitude conversion factor $(A)$ and adding an offset. $C$, With units of MSCT events per synapse per 0.8 sec bin to best fit the MSCT data: for the average NMDA current per trial, $A_{\mathrm{NMDA}}=0.0023, C_{\mathrm{NMDA}}=-0.007$; for the average variance in AMPAR current per trial, $A_{\text {variance }}=0.000859, C_{\text {variance }}=0.007$; for the average mEPSC number per 100 msec bin, $A_{\text {mEPSC }}=0.0859, C_{\text {mEPSC }}=0.005$. The AMPAR mEPSC frequency plots and the variance plots deviate at early time points because of event overlap leading to a underrepresentation of events by direct $\mathrm{mEPSC}$ detection at early time points. The zero point is at $50 \mathrm{msec}$ after the last pulse of train stimulus. B, Close-up of the graph in $A$ plotted on a linear scale.

AMPAR mEPSC frequency by a conversion factor and added a small offset (ranging from 14 to $19 \%$ of the average MSCT rate) (Fig. 4, legend). Given that these measures can involve different recording conditions with different sources of noise and that some error is apparent in MSCT frequency determination, these small offsets are acceptable.

Over a 5-16 sec period after train stimulation, the frequency 
of AMPAR mEPSC appearance decayed with a time course similar to that observed for MSCT frequency. Assuming that the MSCTs appeared with a time course determined by fitting of asynchronous release rates (measured by the AMPAR mEPSCs), we calculated a peak rate of 21 MSCTs per second per synapse ( $Y$-intercept 21 events per second per synapse, adjusted for a 0.8 sec bin). Based on electrophysiological data obtained using these conditions, the peak MSCT rate would correspond to $\sim 2.1 \pm 0.2$ RRPs per second. Therefore, we estimated that the RRP should contain $\sim 10$ quanta (21 MSCTs per second per 2.1 RRPs per second) assuming that an MSCT is mediated by a single quantum. To check the results with another measurement of mEPSC event frequency, we also plotted the decay of average NMDAR current (for a $0.8-17 \mathrm{sec}$ period after the train) measured during the actual imaging experiments and found that it decayed with a slow time course that approximated that of the MSCT rates: $4010 \pm 470 \mathrm{msec}(n=5$ cells) (Fig. 4$)$. Given that individual NMDAR EPSCs decayed with a time constant of $<100 \mathrm{msec}$ in these cells, at time points much longer than the EPSC decay time course (three or more time constants), the time course of the NMDAR current should approximate the decay of mEPSC rate. We did not plot the NMDAR current at earlier time points $(<0.8$ sec after train stimulation) because it is relatively inaccurate for several reasons: (1) NMDAR mEPSCs can be long lasting (up to several hundred milliseconds, in particular for extrasynaptic currents after trains), and their time course would not necessarily provide a measure of release rates but would reflect receptor biophysics (Dingledine et al., 1999), and (2) at early time points the NMDAR current time course also reflects the washout of the antagonist D-AA ( $\sim 400 \mathrm{msec}$ ) and its unbinding from the receptor; see slow rise of current after solution change (Fig. $2 \mathrm{~B}$ ). Thus, although $\mathrm{Ca}^{2+}$ imaging provides us with rates of postsynaptic activity at single synapses, it does not provide us with postsynaptic measures of release rates at early time points after a stimulus train $(<5 \mathrm{sec})$. By using electrophysiological measures we can estimate relative changes in mini-rates (MSCTs) for periods in which direct measurements using imaging were not technically possible. In this case agreement between multiple measures of postsynaptic activity indicative of mEPSC frequency, such as NMDAR current and AMPAR mEPSC frequency, indicate that relative changes in MSCT rates well after a train can be used to extrapolate to peak rates just after train stimulation.

\section{The relationship between evoked release probability and asynchronous release}

Several reports have shown that the evoked release probability $\left(P_{\mathrm{r}}\right)$ (mediated by APs) is heterogeneous between terminals, even those derived from a single axon (Hessler et al., 1993; Rosenmund et al., 1993; Murthy et al., 1997; Chavis and Westbrook, 2001). The presence of heterogeneity in $P_{\mathrm{r}}$ raises the possibility that synapses undergoing asynchronous release may not be representative of the general population. Possibly, synapses exhibiting a high asynchronous release rate do so because they have a relatively low $P_{\mathrm{r}}$ and a large store of quanta and thus are resistant to depression produced by depletion of transmitter stores (Sakaba and Neher, 2001). To investigate whether $P_{\mathrm{r}}$ and asynchronous release rate are correlated at autaptic synapses, our strategy has been to compare the use-dependent block of NMDAR EPSCs by MK-801 as a measure of $P_{\mathrm{r}}$ under control conditions and after previously blocking 50\% of the NMDARs that are activated by asynchronous release with MK-801. Presumably if synapses that undergo asynchronous release had a significantly different $P_{\mathrm{r}}$ (Chavis and Westbrook, 2001) or
NMDAR composition (Nimchinsky et al., 2004), then we would expect the rate of EPSC block to be altered if $50 \%$ of the NMDARs were previously blocked using an MK-801 treatment that selectively targets asynchronous transmission (Fig. 5).

To isolate NMDARs undergoing asynchronous release (allowing their selective blockade by MK-801), we used train stimulation (100 stimuli at $20 \mathrm{~Hz}$ ) that was found previously to completely block evoked (phasic) release, leaving only asynchronous release after $\sim 20$ or 30 stimuli (Otsu et al., 2004) (Fig. 5Ba). During the phase of synaptic transmission in which only asynchronous release was present (Fig. $5 A, B b$ ), we switched to a $\mathrm{Mg}^{2+}$-free solution containing blockers of AMPARs as well as 10 $\mu \mathrm{M}$ glycine (conditions that isolate and augment NMDAR responses). Under these conditions we observed postsynaptic NMDAR currents attributed to asynchronous release. During this period of NMDAR-dependent asynchronous release, we applied MK-801 during multiple trials (one to seven) of $20 \mathrm{~Hz}$ train stimulation (Fig. 5A) (train/MK-801 experiments). This antagonist treatment resulted in $\sim 50 \%$ reduction in the amplitude of evoked NMDAR EPSCs (determined after recovery from depression) (Fig. $5 B b, C$ ). To control for possible train-dependent depression mediated by other means, we also examined trials of train stimulation without MK-801. In these experiments (train experiments), we actually observed a modest potentiation of NMDAR EPSCs (124.8 $\pm 8.0 \%$ ) (Fig. $5 C$ ) and a modest depression of AMPAR EPSCs (Fig. 5 C). In trials performed in the presence of MK-801, no additional reduction in the AMPAR EPSC was observed, whereas the NMDAR EPSC was markedly reduced (Fig. 5C) (compared with train experiments).

We then assessed the impact of blocking a large proportion of synapses that undergo asynchronous release $(\sim 50 \%$ block with MK-801) on a subsequent determination of $P_{\mathrm{r}}$, again using usedependent MK-801 block ( $0.1 \mathrm{~Hz}$ low-frequency stimulation) under conditions designed to block phasic release-generated NMDAR EPSCs (Fig. 5Ba,D) as in previous studies (Chavis and Westbrook, 2001). Previous work indicates that the rapid depression of NMDAR EPSCs by MK-801 corresponds to high $P_{\mathrm{r}}$ synapses that the antagonist can preferentially access and block (Hessler et al., 1993; Rosenmund et al., 1993; Chavis and Westbrook, 2001). Although synapses with a lower $P_{\mathrm{r}}$ have a slower rate of MK-801 block because they are less likely to open and be accessed by the use-dependent antagonist, it is also possible that differences in NMDAR composition and receptor saturation between synapses may result in altered NMDAR blocking rate by MK-801 (Nimchinsky et al., 2004). Consistent with previous investigators using MK-801 treatment, we observed a biexponential depression of evoked synaptic transmission during low-frequency stimulation $(0.1 \mathrm{~Hz})$ with MK-801 (Fig. 5D). We next assessed whether blockade of 50\% of NMDARs in synapses undergoing asynchronous release would affect the MK-801 blocking rate for phasic release. Examination of individual experiments indicated no significant change in the rate of the MK-801 block (analysis of weighted time constants $p>0.05$, by unpaired $t$ test; train $3.1 \pm 0.2$ and train/MK-801 $3.4 \pm 0.2$ ) for cells in which $\sim 50 \%$ synaptic NMDARs undergoing asynchronous release were blocked previously by MK-801 or their controls $(n=$ 7-12). To better assess whether the proportion of synapses with fast and slow MK-801-mediated blockade $(0.1 \mathrm{~Hz}$ stimulation, indicative of high and low $P_{\mathrm{r}}$ ) changed, we used normalized average data (across different cells) to determine whether the proportion of the decay described by a slow component changed. Previous data (Chavis and Westbrook, 2001) and our own (Fig. 6) indicated that if high $P_{\mathrm{r}}$ synapses are blocked, the proportions 
A

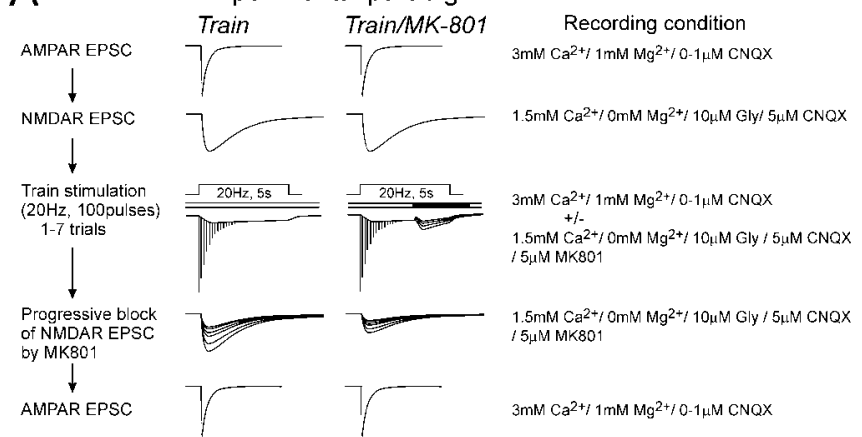

$\mathrm{Ba}$

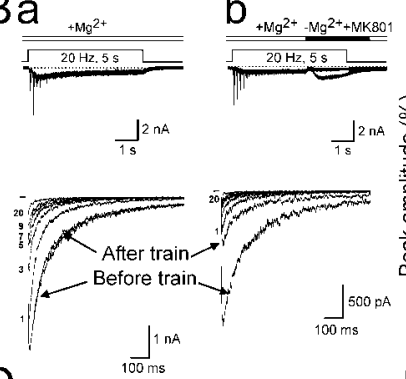

D
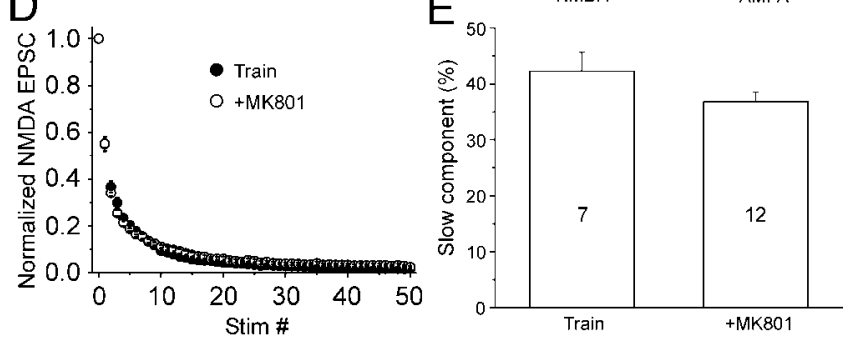

Figure 5. The use of partial MK-801 block to determine whether synapses undergoing asynchronous release belong to a unique population. $A$, A schematic of the experiments to monitor use-dependent blockade of NMDAR EPSCS. AMPAREPSC was monitored before and after recording NMDAR EPSC and train stimulation to confirm a postsynaptic effect of MK-801. NMDAR EPSCs were monitored under $1.5 \mathrm{~mm} \mathrm{Ca}^{2+} / 0 \mathrm{~mm} \mathrm{Mg}^{2+}, 10 \mu \mathrm{m}$ glycine, and $5 \mu \mathrm{m}$ CNQX. In train experiments, after monitoring NMDAR EPSCs the autaptic cell was stimulated with one to seven trials of tetanic stimulation $\left(5 \mathrm{sec}\right.$ of $20 \mathrm{~Hz}$ ) at $1-2$ min intervals under $3 \mathrm{~mm} \mathrm{Ca}{ }^{2+} / 1 \mathrm{~mm}$ $\mathrm{Mg}^{2+}$ and $0-1 \mu \mathrm{M}$ CNQX (0 or $1 \mu \mathrm{M}$ CNQX was used at times to reduce EPSC amplitude to improve voltage clamp). After this stimulation, progressive blockade of NMDAR EPSCs by $5 \mu \mathrm{m}$ MK-801 (stimulation at $0.1 \mathrm{~Hz}$ ) was used to assess $P_{r}$. Train/MK-801 experiments were the same as in train experiments, except that NMDAR EPSCs were partially blocked during conditioning trains. In alternating trains, the extracellular solution was switched to one in which NMDAR activity was facilitated ( $0 \mathrm{~mm} \mathrm{Mg}{ }^{2+}$ and $10 \mu \mathrm{m}$ glycine) and subject to a use-dependent manner using MK-801. Ba, An example of train experiments. After monitoring NMDAR EPSCs (Before train; bottom), the autaptic cell was stimulated with four trials of tetanic stimulation ( 5 sec of 20 $\mathrm{Hz}$ ) at 1 min intervals (top). During the tetanic stimulation, phasic release was completely blocked after $\sim 2.5 \mathrm{sec}$. Continuing for several seconds after the train was a period with enhanced asynchronous transmission. The record shown was made under conditions during which AMPA receptor activity was readily visible (no CNQX). After the four conditioning trains the NMDAR EPSC was monitored under the same condition as before the tetanic stimulation (After train; bottom). During stable NMDAR EPSC amplitude (stimulation at $0.1 \mathrm{~Hz}$ ), $5 \mu \mathrm{m}$ MK-801 was added to the extracellular solution and progressive blockade of NMDAR EPSCs was used to assess $P_{r}$. Five traces are superimposed and shown by stimulus number after addition of MK-801 (bottom). Bb, An example of train/MK-801 experiments. Three seconds after tetanic stimulation the extracellular solution including $\mathrm{Mg}^{2+}$ was changed to one with $0 \mathrm{Mg}^{2+}$ and 5 $\mu \mathrm{M}$ MK-801 for $3 \mathrm{sec}$ with computer-controlled solenoid-driven valves. Three overplotted trials show progressing blockade of NMDARs during asynchronous release (top). NMDAR EPSCs before and after the conditioning trains and five during progressive blockade by MK-801 application are superimposed (bottom). C, Ratio of NMDAR EPSC amplitude after to before the conditioning trains (20 Hzfor 5 secstimulation) in the presence (+MK-801) or in the absence of MK-801 (Train) are plotted in two columns at the left. Ratio of AMPAR EPSC amplitude after to before the conditioning trains and progressive blockade of NMDAR EPSCs by MK-801 in two columns at the right (Train or + MK-801). The numbers in the bars show cell number. D, Relative progressive-blockade of NMDAR EPSCs by MK-801 after conditioning trains were delivered with (open circle; $n=12$ cells) and without MK-801

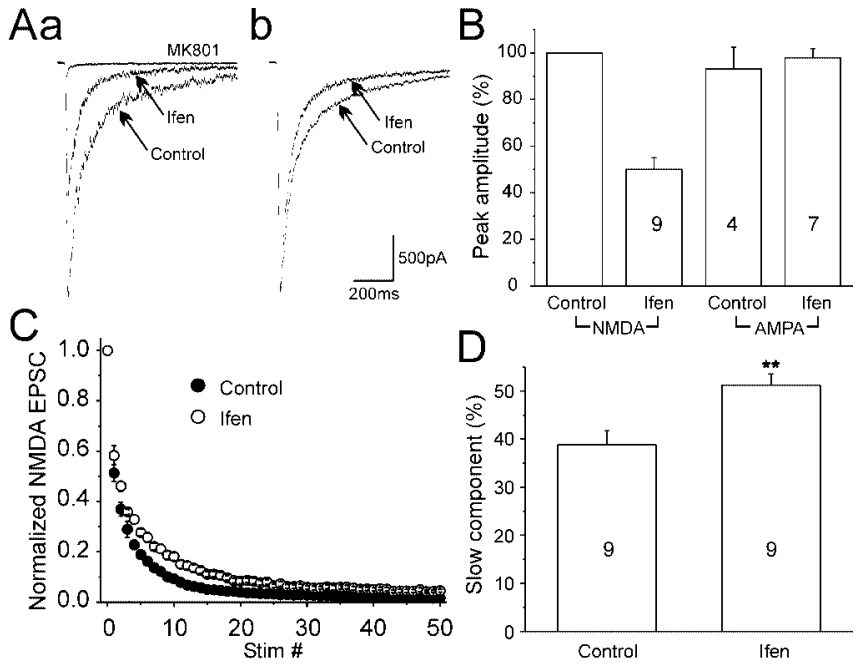

Figure 6. Ifenprodil blocks high $P_{\mathrm{r}}$ synapses and reveals two components of use-dependent MK-801 block. Aa, Representative traces of NMDAR EPSCs under control conditions $(0 \mathrm{~mm}$ $\mathrm{Mg}^{2+}, 10 \mu \mathrm{m}$ glycine, and $5 \mu \mathrm{m}$ (NQX) and addition of $3 \mu \mathrm{m}$ ifenprodil. The EPS(s were mediated entirely by NMDA receptors because progressive blockade by MK-801 (at 70th stimulus after ifenprodil application) could completely block them. Stimulus artifacts are removed. $A b$, Normalized EPSCS recorded under control conditions or ifenprodil reveal that ifenprodil selectively blocks the slower component of EPSC. B, Ratio of NMDAR EPSC amplitude after to before ifenprodil treatment (Ifen) is plotted in second column at the left. Ratio of AMPAR EPSC amplitude after to before either ifenprodil treatment (Ifen) or nontreatment (Control) and progressive blockade of NMDAR EPSCS by MK- 801 are plotted in two columns at the right. The numbers in the bars show cell number. C, Use-dependent blockade of NMDAR EPSCs by MK-801 ( $0.1 \mathrm{~Hz}$ stimulation). Antagonism of NR2B-containing NMDARs by ifenprodil leads to a change in the blocking rate for the EPSC by MK-801 (open circle; $n=9$ ) compared with nontreatment condition (filled circle; $n=9$ ). NMDAR EPSC amplitude was normalized to responses just before application of MK-801. Data shown are the mean \pm SEM. D, The result indicates a significant increase in the slow component of block with ifenprodil treatment $(p<0.01)$. The fitting procedure for EPSC use-dependent block by MK-801 is described in Figure $5 D$.

of fast and slow MK-801 block should change (slow component should increase), and not necessarily the time constants. The average time constants for cells without MK-801 application during conditioning $100 \mathrm{~Hz}$ train stimulation was $0.72 \pm 0.09$ stimuli for the fast component and $6.17 \pm 0.60$ stimuli for the slow component $(n=9)$. Therefore, to increase statistical power we constrained the fitting by assuming an average fast and slow time constant derived from control trains and determined whether the fraction of block mediated by the slow component changed by blocking a large fraction of NMDARs during asynchronous release. Using this analysis, no significant change in the percentage of slow component MK-801 block was observed after blocking

(filled circle; $n=7$ cells) to block a proportion of synapses undergoing asynchronous release. NMDAR EPSC amplitudes were normalized to the response taken just before application of MK-801. The data shown is the mean \pm SEM. E, The data from individual cells (average of all cells shown in () was fitted to a model constrained by fast ( 0.72 stimuli) and slow ( 6.17 stimuli) time constants. These values are averages resulting from the bi-exponential fitting of progressive blockade of NMDAR EPSCs by MK-801 in each cell in a control experiment in Figure $6(n=$ 9). The fitting procedure indicated no significant difference in the slow component of NMDAR EPSC block mediated by MK-801, whether conditioning trains were given to remove some synapses undergoing high asynchronous release rates or not. Graph shows the percentage of use-dependent EPSC blockade by MKI-801 mediated by a slow component for neurons in which $20 \mathrm{~Hz}(5 \mathrm{sec})$ conditioning trains were given in the absence (Train; $n=7$ ) or presence (MK-801; $n=12$ ) of MK-801 to partially block NMDARs activated during asynchronous release. No significant change in the slow component was observed when $\sim 50 \%$ of the NMDARs undergoing asynchronous release were blocked. 
$\sim 50 \%$ of the NMDARs with MK-801 during $100 \mathrm{~Hz}$ traininduced asynchronous release (Fig. $5 E)(p>0.05)$. If the synapses that were blocked during the asynchronous release were of relatively higher $P_{\mathrm{r}}$, we would expect a higher proportion of low probability synapses when use-dependent MK-801 block of phasic release was performed (low-frequency stimulation, $0.1 \mathrm{~Hz}$ ). Our results, however, showed that there was no correlation between the asynchronous release rate and $P_{\mathrm{r}}$ because blockade of $50 \%$ of synaptic NMDARs by MK- 801 application during the asynchronous phase of release had no effect on subsequent measurement of $P_{\mathrm{r}}$ using low-frequency stimulation (Fig. 5D). These findings are consistent with synapses that undergo high rates of asynchronous release being drawn from a general population and not reflecting a subset of synapses that have high or low $P_{\mathrm{r}}\left(P_{\mathrm{r}}\right.$ defined by MK- 801 block during phasic release, $0.1 \mathrm{~Hz}$ stimulation) or markedly different NMDAR composition.

The results raise the question of whether a small difference in $P_{\mathrm{r}}$ could be measured with our technique. Previous work by Chavis and Westbrook (2001) indicate that blockers of NMDARs containing NR1/NR2B subunits such as ifenprodil can be used to selectively reduce the postsynaptic contribution of high $P_{\mathrm{r}}$ synapses. This effect is because two populations of synapses are thought to exist and not because of a potential presynaptic effect of ifenprodil. We confirmed that high $P_{\mathrm{r}}$ synapses preferentially have NR1/NR2B NMDARs as shown by Chavis and Westbrook (2001). EPSCs resulting from NR2B-containing NMDARs have been shown to have a slower decay time constant than those arising from NR2A NMDARs (Hessler et al., 1993) (Fig. 6A). In the presence of the NR1/NR2B blocker ifenprodil, we observed that NMDAR EPSCs decayed more rapidly $(64.8 \pm 0.6 \mathrm{msec}$; ifenprodil, $n=9$; vs $97.5 \pm 4.5 \mathrm{msec}$ control; $p<0.01$ paired $t$ test) (Fig. 6A). Ifenprodil treatment was relatively selective to postsynaptic sites (NMDARs) because it has no effect on AMPAR EPSCs (Fig. 6B). In the presence of ifenprodil using the same analysis as described for Figure 5, we found a significant increase in the slow component of MK-801 blocking rate during lowfrequency phasic release (Fig. $6 C, D$ ). With ifenprodil present we propose that high $P_{\mathrm{r}}-\mathrm{NR} 1 / \mathrm{NR} 2 \mathrm{~B}$ bearing synapses are antagonized (Fig. $6 A, B$ ) and prevented from participating in usedependent block by MK-801, leading to an apparently lower $P_{\mathrm{r}}$ that is reflected in the reduced $\mathrm{MK}-801$ blocking rate. In total these experiments, which show expected changes in $P_{\mathrm{r}}$ by blocking high $P_{\mathrm{r}}$ synapses with ifenprodil but no change in $P_{\mathrm{r}}$ associated with synapses undergoing asynchronous release, support the proposal that NMDARs undergoing asynchronous release do not represent a unique group.

\section{Discussion}

\section{Postsynaptic determination of rate release at individual synapses during train stimulation}

Our results provide estimates for peak rates of exocytosis at CNS synapses by imaging the postsynaptic effect of asynchronous release. We estimate that hippocampal synapses are able to release $\sim 21$ quanta per second at steady state during trains. These results suggest that release must be maintained at these rates by rapid mechanisms and not necessarily continuous endocytosis and clathrin-mediated recycling of vesicles that elegant studies from the neuromuscular junction estimate to require tens of seconds to occur (Cochilla et al., 1999; Richards et al., 2000; Harata et al., 2001b). Recent studies imaging the stimulus evoked turnover of clathrin in hippocampal neurons suggest that it exhibits delayed kinetics (Mueller et al., 2004) that cannot account for the high peak rates of release that we observe. Given that the observed high rates of asynchronous release can be maintained for only a few seconds, it is likely that they reflect not a recycling mechanism but refilling from a reserve pool of finite size. Perhaps $\mathrm{Ca}^{2+}$ dependent mechanisms engaged during train stimulation contribute to these high rates of refilling (Stevens and Wesseling, 1998; Wang and Kaczmarek, 1998). Rates of quantal release and vesicle turnover are temperature dependent and likely to be higher at physiological temperature than room temperature $\left(\sim 21^{\circ} \mathrm{C}\right)$ as we have done (Pyott and Rosenmund, 2002; Fernandez-Alfonso and Ryan, 2004); however, recent work indicates that the $20 \mathrm{~Hz}$ trains that we use produce depression at $37^{\circ} \mathrm{C}$ (Fernandez-Alfonso and Ryan, 2004).

\section{Potential sources of error}

One limitation of this approach is that we cannot actually resolve individual MSCTs during the peak rate of asynchronous release. There are several concerns with measuring MSCTs during early periods after train stimulation. (1) $\mathrm{Ca}^{2+}$ indicator saturation induces nonlinearity to $\mathrm{Ca}^{2+}$ signaling, and (2) many double and triple events can occur over the imaging sampling time $(\sim 66$ msec). Because of this limitation and missed events, the decay of MSCT rates may not strictly follow a single exponential. To get at the issue of missed events, we took an average fluorescent signal from a large number of individual dendritic sites with and without the removal of NMDA receptor blockers after train stimulation. Under these conditions, we observed a large and longlasting NMDAR-dependent $\mathrm{Ca}^{2+}$ transient, indicating that many early MSCT events (at times $<5 \mathrm{sec}$ ) were likely missed. Another problem with imaging MSCTs just after train stimulation is that antagonist must be applied to the entire neuron, limiting the solution exchange times to no faster than several hundred milliseconds. For this reason the NMDAR current triggered by asynchronous release that we measure does not reflect the peak rate of quantal release as assayed postsynaptically. To better assess the peak rates of asynchronous activity, we have relied on monitoring AMPAR activity that does not have the limitation of slow receptor kinetics or the requirement to switch solutions rapidly (to wash out NMDAR antagonists). By combining several measures of AMPAR activity with NMDAR currents at later time points, we are confident that significant rates of asynchronous release continue for up to $10 \mathrm{sec}$ after stimulation, and these rates correlate with peak activity just after train stimulation. Given that we have used $400 \mu \mathrm{M}$ Fluo- 4 as our $\mathrm{Ca}^{2+}$ indicator, it is conceivable that the asynchronous release that we observe may be blunted in peak rate and prolonged in time course because Fluo-4 will buffer $\mathrm{Ca}^{2+}$ with characteristics determined by its $\mathrm{Ca}^{2+}$ binding kinetics and concentration (Helmchen and Tank, 2000); however, it is not really possible to monitor the presynaptic concentration of Fluo-4 in terminals. Clearly, the use of conventional synapses with separate presynaptic and postsynaptic compartments (instead of autapses) would be a way to address this concern in future studies. In studies with separate presynaptic and postsynaptic compartments, presynaptic buffering capacity could even be tuned to vary the time course of asynchronous release.

\section{Using partial MK-801 to assess the impact of synapses undergoing asynchronous release on evoked $P_{r}$}

The application of use-dependent MK- 801 block indicated that synapses undergoing asynchronous release were indistinguishable from the general population with regard to their $P_{\mathrm{r}}$. Evidence for this included no significant change in the MK-801 blocking rate during phasic release (triggered by $0.1 \mathrm{~Hz}$ stimulation) when 
$50 \%$ of synaptic NMDAR was previously removed by MK-801 treatment designed to target asynchronous release; however, the results also raise the question of whether the technique is sensitive enough to resolve small changes in $P_{\mathrm{r}}$. Plotting the average time courses of phasic release block by MK-801 for cases with and without $50 \%$ of the NMDARs participating in asynchronous release blocked indicated that the datasets were virtually superimposable. To assess any statistical change we relied on fitting these plots to a model with two populations of synapses, either high or low $P_{\mathrm{r}}$ or perhaps varied NMDAR composition or saturation by agonist. It is conceivable that this is an oversimplification, but as mentioned above, simple inspection of the data reveals no major effect. Importantly, positive controls support the two-population statistical model in that ifenprodil treatment, which blocks high-probability synapses, produces a significant change in the time course of the MK- 801 blocking rate that can be modeled by an increase in a slow component of block.

\section{Postsynaptic measures of quantal release after train stimulation}

Postsynaptic $\mathrm{Ca}^{2+}$ imaging complements monitoring presynaptic vesicle release rate and provides additional information. First, we are monitoring actual synaptic responses and not membrane recycling, which may not always reflect transmitter release. Second, the results draw attention to the fact that biochemical events triggered by $\mathrm{Ca}^{2+}$ influx continue to occur many seconds after train stimulation. Perhaps these continued biochemical signals mediated by mEPSCs elicit specific forms of signal transduction (Otsu and Murphy, 2003a,b). Imaging asynchronous release may also represent a technique to gain insight into the workings of the synapse during high-frequency stimulation when most electrophysiological approaches would be unable to resolve individual quanta. It could be argued that some of our experiments could be repeated with FM dyes; however, given that asynchronous release closely follows phasic release, it would be difficult to apply FM dyes in such a manner that they would selectively label terminals undergoing asynchronous release and not terminals showing delayed dye uptake triggered by phasic release. Previous investigators have examined the response of spines to high-frequency stimulation and observed persistent changes in spine $\left[\mathrm{Ca}^{2+}\right]_{\mathrm{i}}$ that may have been related in part to asynchronous release (Muller and Connor, 1991); however, the sampling rates used were not high enough to resolve individual quanta. By using extrapolation and monitoring the rates of asynchronous release, it is possible to image a reflection of high-frequency signaling that occurs during train stimulation. This high-frequency signaling is particularly relevant considering that plastic behaviors are often associated with high-frequency gamma and theta rhythmic activity in vivo (Buzsaki et al., 2002). If high-frequency presynaptic activity at a single terminal is prolonged for several seconds, phasic release could be depressed and marked asynchronous release would be apparent. Recent experiments using cultured hippocampal neurons suggest that synaptic transmission can be maintained presynaptically with up to $10 \mathrm{~Hz}$ stimulation at physiological temperature (Fernandez-Alfonso and Ryan, 2004). These authors propose that this occurs through rapid mechanisms of endocytosis and vesicle recycling, although they did not directly resolve the postsynaptic action of individual release events.

\section{Comparison of asynchronous release measures of transmitter release with other studies}

Previously, investigators using FM dye imaging, electrophysiological techniques combined with local application of agents to stimulate synaptic release, have estimated the RRP size and rate of quantal release at individual pyramidal neuron synapses (Liu and Tsien, 1995a,b; Stevens and Tsujimoto, 1995; Murthy and Stevens, 1999; Schikorski and Stevens, 2001). Our present results using a different approach that does not necessarily rely on stimulating only individual synapses with an extracellularly applied agent or monitoring potentially indirect vesicle recycling as a measure of release now suggest similar peak rates of quantal release. In total, our results and others indicate that CNS synapses are able to undergo rapid bursts of transmission averaging $\sim 20$ quanta per second. The ability of the synapse to sustain this level of release will likely be dependent on the size of its RRP and the rate at which it can refill from a reserve pool, reuse quanta via kiss and run, or recycle vesicles via clathrin-mediated endocytosis. Elegant recent electron microscopy data from the frog neuromuscular junction (Rizzoli and Betz, 2004) indicate that recycling vesicles belonging to the RRP may actually be distributed throughout the synapse, suggesting that refilling processes may not be constrained by the number of vesicles resident at plasma membrane release sites. Given the potential for transitions in release mechanisms (Aravanis et al., 2003; Gandhi and Stevens, 2003), future studies will require investigations during which both presynaptic vesicle trafficking and the postsynaptic quantal response can be monitored simultaneously at an identified synapse.

\section{References}

Abdul-Ghani MA, Valiante TA, Pennefather PS (1996) $\mathrm{Sr}^{2+}$ and quantal events at excitatory synapses between mouse hippocampal neurons in culture. J Physiol (Lond) 495:113-125.

Aravanis AM, Pyle JL, Tsien RW (2003) Single synaptic vesicles fusing transiently and successively without loss of identity. Nature 423:643-647.

Atluri PP, Regehr WG (1998) Delayed release of neurotransmitter from cerebellar granule cells. J Neurosci 18:8214-8227.

Bekkers JM, Stevens CF (1991) Excitatory and inhibitory autaptic currents in isolated hippocampal neurons maintained in cell culture. Proc Natl Acad Sci USA 88:7834-7838.

Buzsaki G, Csicsvari J, Dragoi G, Harris K, Henze D, Hirase H (2002) Homeostatic maintenance of neuronal excitability by burst discharges in vivo. Cereb Cortex 12:893-899.

Chavis P, Westbrook G (2001) Integrins mediate functional pre- and postsynaptic maturation at a hippocampal synapse. Nature 411:317-321.

Cochilla AJ, Angleson JK, Betz WJ (1999) Monitoring secretory membrane with FM1-43 fluorescence. Annu Rev Neurosci 22:1-10.

Cummings DD, Wilcox KS, Dichter MA (1996) Calcium-dependent paired-pulse facilitation of miniature EPSC frequency accompanies depression of EPSCs at hippocampal synapses in culture. J Neurosci 16:5312-5323.

Dingledine R, Borges K, Bowie D, Traynelis SF (1999) The glutamate receptor ion channels. Pharmacol Rev 51:7-61.

Dobrunz LE, Stevens CF (1997) Heterogeneity of release probability, facilitation, and depletion at central synapses. Neuron 18:995-1008.

Emptage NJ, Reid CA, Fine A, Bliss TV (2003) Optical quantal analysis reveals a presynaptic component of LTP at hippocampal Schafferassociational synapses. Neuron 38:797-804.

Fernandez-Alfonso T, Ryan TA (2004) The kinetics of synaptic vesicle pool depletion at CNS synaptic terminals. Neuron 41:943-953.

Gandhi SP, Stevens CF (2003) Three modes of synaptic vesicular recycling revealed by single-vesicle imaging. Nature 423:607-613.

Harata N, Ryan TA, Smith SJ, Buchanan J, Tsien RW (2001a) Visualizing recycling synaptic vesicles in hippocampal neurons by FM 1-43 photoconversion. Proc Natl Acad Sci USA 98:12748-12753.

Harata N, Pyle JL, Aravanis AM, Mozhayeva M, Kavalali ET, Tsien RW (2001b) Limited numbers of recycling vesicles in small CNS nerve terminals: implications for neural signaling and vesicular cycling. Trends Neurosci 24:637-643.

Helmchen F, Tank DW (2000) A single-compartment model of calcium dynamics in nerve terminals and dendrites. In: Imaging neurons: a labo- 
ratory manual (Yuste R, Lanni F, Konnerth A, eds), pp 33.1-33.11. Cold Spring Harbor, NY: Cold Spring Harbor Laboratory.

Hessler NA, Shirke AM, Malinow R (1993) The probability of transmitter release at a mammalian central synapse. Nature 366:569-572.

Holthoff K, Tsay D, Yuste R (2002) Calcium dynamics of spines depend on their dendritic location. Neuron 33:425-437.

Katz B, Miledi R (1972) The statistical nature of the acetylcholine potential and its molecular components. J Physiol (Lond) 224:665-699.

Klingauf J, Kavalali ET, Tsien RW (1998) Kinetics and regulation of fast endocytosis at hippocampal synapses. Nature 394:581-585.

Li B, Chen N, Luo T, Otsu Y, Murphy TH, Raymond LA (2002) Differential regulation of synaptic and extra-synaptic NMDA receptors. Nat Neurosci 5:833-834.

Liu G, Tsien RW (1995a) Synaptic transmission at single visualized hippocampal boutons. Neuropharmacology 34:1407-1421.

Liu G, Tsien RW (1995b) Properties of synaptic transmission at single hippocampal synaptic boutons. Nature 375:404-408.

Mackenzie PJ, Umemiya M, Murphy TH (1996) $\mathrm{Ca}^{2+}$ imaging of CNS axons in culture indicates reliable coupling between single action potentials and distal functional release sites. Neuron 16:783-795.

Mackenzie PJ, Kenner GS, Prange O, Murphy TH (2000) Vesicle number does not predict postsynaptic measures of miniature synaptic activity frequency in cultured cortical neurons. Neuroscience 98:1-7.

Mainen ZF, Malinow R, Svoboda K (1999) Synaptic calcium transients in single spines indicate that NMDA receptors are not saturated. Nature 399:151-155.

Mueller VJ, Wienisch M, Nehring RB, Klingauf J (2004) Monitoring clathrin-mediated endocytosis during synaptic activity. J Neurosci 24:2004-2012.

Muller W, Connor JA (1991) Dendritic spines as individual neuronal compartments for synaptic $\mathrm{Ca}^{2+}$ responses. Nature 354:73-76.

Murphy TH, Baraban JM, Wier WG (1995) Mapping miniature synaptic currents to single synapses using calcium imaging reveals heterogeneity in postsynaptic output. Neuron 15:159-168.

Murthy VN, Stevens CF (1999) Reversal of synaptic vesicle docking at central synapses. Nat Neurosci 2:503-507.

Murthy VN, Sejnowski TJ, Stevens CF (1997) Heterogeneous release properties of visualized individual hippocampal synapses. Neuron 18:599-612.

Murthy VN, Sejnowski TJ, Stevens CF (2000) Dynamics of dendritic calcium transients evoked by quantal release at excitatory hippocampal synapses. Proc Natl Acad Sci USA 97:901-906.

Neher E, Augustine GJ (1992) Calcium gradients and buffers in bovine chromaffin cells. J Physiol (Lond) 450:273-301.

Nimchinsky EA, Yasuda R, Oertner TG, Svoboda K (2004) The number of glutamate receptors opened by synaptic stimulation in single hippocampal spines. J Neurosci 24:2054-2064.

Oertner TG, Sabatini BL, Nimchinsky EA, Svoboda K (2002) Facilitation at single synapses probed with optical quantal analysis. Nat Neurosci 5:657-664.

Otsu Y, Murphy TH (2003a) Miniature transmitter release: accident of nature or careful design? Sci STKE 211:pe54.
Otsu Y, Murphy TH (2003b) Mind-altering miniature neurotransmitter release? Proc Natl Acad Sci USA 100:5589-5590.

Otsu Y, Shahrezaei V, Li B, Raymond LA, Delaney KR, Murphy TH (2004) Competition between phasic and asynchronous release for recovered synaptic vesicles at developing hippocampal autaptic synapses. J Neurosci 24:420-433.

Pyle JL, Kavalali ET, Piedras-Renteria ES, Tsien RW (2000) Rapid reuse of readily releasable pool vesicles at hippocampal synapses. Neuron 28:221-231.

Pyott SJ, Rosenmund C (2002) The effects of temperature on vesicular supply and release in autaptic cultures of rat and mouse hippocampal neurons. J Physiol (Lond) 539:523-535.

Raju B, Murphy E, Levy LA, Hall RD, London RE (1989) A fluorescent indicator for measuring cytosolic free magnesium. Am J Physiol 256:C540-C548.

Richards DA, Guatimosim C, Betz WJ (2000) Two endocytic recycling routes selectively fill two vesicle pools in frog motor nerve terminals. Neuron 27:551-559.

Rizzoli SO, Betz WJ (2004) The structural organization of the readily releasable pool of synaptic vesicles. Science 303:2037-2039.

Rosenmund C, Stevens CF (1996) Definition of the readily releasable pool of vesicles at hippocampal synapses. Neuron 16:1197-1207.

Rosenmund C, Clements JD, Westbrook GL (1993) Nonuniform probability of glutamate release at a hippocampal synapse. Science 262:754-757.

Ryan TA, Reuter H, Wendland B, Schweizer FE, Tsien RW, Smith SJ (1993) The kinetics of synaptic vesicle recycling measured at single presynaptic boutons. Neuron 11:713-724.

Sabatini BL, Oertner TG, Svoboda K (2002) The life cycle of $\mathrm{Ca}^{2+}$ ions in dendritic spines. Neuron 33:439-452.

Sakaba T, Neher E (2001) Calmodulin mediates rapid recruitment of fastreleasing synaptic vesicles at a calyx-type synapse. Neuron 32:1119-1131.

Sara Y, Mozhayeva MG, Liu X, Kavalali ET (2002) Fast vesicle recycling supports neurotransmission during sustained stimulation at hippocampal synapses. J Neurosci 22:1608-1617.

Schikorski T, Stevens CF (2001) Morphological correlates of functionally defined synaptic vesicle populations. Nat Neurosci 4:391-395.

Stevens CF, Tsujimoto T (1995) Estimates for the pool size of releasable quanta at a single central synapse and for the time required to refill the pool. Proc Natl Acad Sci USA 92:846-849.

Stevens CF, Wesseling JF (1998) Activity-dependent modulation of the rate at which synaptic vesicles become available to undergo exocytosis. Neuron 21:415-424.

Umemiya M, Senda M, Murphy TH (1999) Behaviour of NMDA and AMPA receptor-mediated miniature EPSCs at rat cortical neuron synapses identified by calcium imaging. J Physiol (Lond) 521:113-122.

Umemiya M, Chen N, Raymond LA, Murphy TH (2001) A calciumdependent feedback mechanism participates in shaping single NMDA miniature EPSCs. J Neurosci 21:1-9.

Wang LY, Kaczmarek LK (1998) High-frequency firing helps replenish the readily releasable pool of synaptic vesicles. Nature 394:384-388.

Wesseling JF, Lo DC (2002) Limit on the role of activity in controlling the release-ready supply of synaptic vesicles. J Neurosci 22:9708-9720. 